\title{
Dielectric Spectroscopy of Proteins as a Quantitative Experimental Test of Computational Models of Their Low-Frequency Harmonic Motions
}

\author{
N. Q. Vinh, ${ }^{*,+\ddagger}$ S. James Allen, ${ }^{+, \neq}$and Kevin W. Plaxco ${ }^{\S}$ \\ ${ }^{\dagger}$ Institute for Terahertz Science and Technology, ${ }^{\ddagger}$ Department of Physics, and ${ }^{\S}$ Department of Chemistry and Biochemistry, \\ University of California, Santa Barbara, Santa Barbara, California 93106, United States
}

Supporting Information

\begin{abstract}
Decades of molecular dynamics and normal mode calculations suggest that the largest-scale collective vibrational modes of proteins span the picosecond to nanosecond time scale. Experimental investigation of these harmonic, low-amplitude motions, however, has proven challenging. In response, we have developed a vector network analyzer-based spectrometer that supports the accurate measurement of both the absorbance and refractive index of solvated biomolecules over the corresponding gigahertz to terahertz frequency regime, thus providing experimental information regarding their largestscale, lowest frequency harmonic motions. We have used this spectrometer to measure the complex dielectric response of lysozyme solutions over the range 65 to $700 \mathrm{GHz}$ and an effective medium model to separate the dielectric response of the solvated protein from that of its buffer. In doing so, we find that each lysozyme is surrounded by a tightly bound layer of $165 \pm 15$ water molecules that, in terms of their picosecond dynamics, behave as if they are an integral part of the protein. We also find that existing computational descriptions of the protein's dynamics compare poorly with the results of our experiment. Specifically, published normal mode and molecular dynamics simulations do not explain the measured dielectric response unless we introduce a cutoff frequency of $250 \mathrm{GHz}$ below which the density of vibrational modes drops to zero. This cutoff is physically plausible, given the known size of the protein and the known speed of sound in proteins, raising questions as to why it is not apparent in computational models of the protein's motions.
\end{abstract}

\section{INTRODUCTION}

Although computational methods suggest that harmonic molecular motions play a vital role in the function of many proteins, ${ }^{1-10}$ these motions remain poorly explored by experiment. For example, while protein motion is thought to play critical roles in substrate binding, catalysis, and sensory transduction cycling, ${ }^{1-3}$ experimental methods of monitoring these structural fluctuations, such as nuclear magnetic resonance (NMR), electron paramagnetic resonance (EPR), and fluorescence spectroscopy, are limited to large amplitude, typically barrier-limited dynamics. ${ }^{11,12}$ In response, recent years have seen significant effort to develop new experimental approaches by which the collective, low amplitude harmonic motions of proteins can be investigated in their native, aqueous environments. ${ }^{6,13,14}$ One approach to this end, gigahertz to terahertz spectroscopy, is predicated on the observation that the relevant global and subglobal harmonic motions occur on the nanosecond to picosecond time scale ${ }^{6,15-19}$ defined by these frequencies. Because proteins are invariably "decorated" with a large number of charges, these collective vibrational modes thus interact strongly with gigahertz to terahertz radiation, suggesting that spectroscopy in this regime can provide insights into the nature of these functionally relevant motions.
Although the above arguments suggest that, in theory, gigahertz to terahertz spectroscopy can provide direct experimental insights into the harmonic motions of biomolecules, in practice several technical challenges render the approach difficult. First among these is the very strong absorption of water over this frequency range, ${ }^{20}$ a hurdle that limited the earliest studies in this field to dry powders or moist solids. ${ }^{621,22}$ Only recently have studies emerged of proteins in their more relevant, solvated states. ${ }^{16-19}$ Even in these experiments, however, the absorbance of the protein was extracted from the measured spectra of protein solutions and the relevant buffer blanks by assuming that the absorbance of the mixture is the weighted average absorbance of its components, an assumption that appears unfounded. Specifically, the true dielectric response (equivalently the absorbance and refractive index) of a mixture is not the weighted sum of the dielectric responses of its components. ${ }^{23}$ Moreover, this problem is particularly acute when the response of the solvent changes very rapidly with frequency, as it does for aqueous solutions below $1 \mathrm{THz}$. This second problem, however, has not yet been addressed in the literature over the frequency range of interest.

Received: January 19, 2011

Published: May 04, 2011 
In order to provide a better description of the dielectric response of mixtures, Maxwell Garnet, Wagner, Bruggeman, and Hanai have developed approximations that, for components orders of magnitude smaller than the wavelengths of the incoming radiation, treat the material as a homogeneous substance with a single, effective-dielectric function. ${ }^{24-27}$ To use these "effective medium approximations" to characterize the dielectric response of protein solutions, however, one must accurately measure both the absorbance and the refractive index of the solution and the equivalent protein-free buffer. Several groups ${ }^{28-30}$ have performed such studies on protein-containing solutions below $20 \mathrm{GHz}$. This frequency regime, however, corresponds to the rotational relaxation of macromolecules and not their internal dynamics. Here we employ a custom-built frequency domain spectrometer to measure the absorbance and refractive index of a protein solution from 65 to $700 \mathrm{GHz}$, which instead captures the protein's collective internal dynamics. We then use these data to directly test computational models of the protein's lowest frequency harmonic motions.

\section{RESULTS AND DISCUSSION}

Here we have studied the gigahertz to terahertz spectroscopy of hen egg white lysozyme, a monomeric protein of molecular weight of $14.3 \mathrm{kDa}$. We employed this protein because it is of known structure and has been the subject of extensive molecular dynamics simulations and normal-mode calculations aimed at elucidating its picosecond-scale motions. We have measured the gigahertz to terahertz dielectric response of lysozyme-containing solutions using a spectrometer based on a vector network analyzer system built by Virginia Diode Inc. This new spectrometer achieves orders of magnitude improved signal-to-noise over previous studies and sub- $100 \mathrm{~Hz}$ spectral resolution over the spectral range from 65 to $700 \mathrm{GHz}(0.43$ to $4.3 \mathrm{~mm}$ or 2.3 to $23.3 \mathrm{~cm}^{-1}$ ). Over this spectral range, the instrument achieves a detection dynamic range spanning more than 11 orders of magnitude, allowing it to accurately measure even very strongly absorbing materials, such as the aqueous samples employed here. Given these attributes, our spectrometer can measure the absorbance of proteins in aqueous solution over concentrations ranging from below $1 \%(\mathrm{w} / \mathrm{w})$ to saturation. Finally, the device can monitor both the magnitude and the phase of the transmitted radiation, thus defining both the absorption and refractive index of the sample.

Using this spectrometer and a variable-thickness cell, ${ }^{18,19,31}$ we have measured the absorbance and phase shift of lysozyme solutions and a matched buffer blank as functions of path length, $d$. Fitting absorbance as a function of path length to Beer's law, $I(d)=I_{0} \exp \left(-\alpha_{\text {sol }} d\right)$, with $I_{0}$ corresponding to the incident intensity, gives us a measurement of the absorption coefficient of the sample, $\alpha_{\text {sol }}$. In parallel we also fit the observed phase shift as a linear function of path length to define $n_{\text {sol }}$, the refractive index of the solution (Figure S1, Supporting Information). Taken together, these data define the absorbance (Figure 1) and refractive index (Figure 1, inset) of lysozyme solutions, both of which are particularly strong functions of frequency in this range, monotonically increasing and decreasing, respectively, with rising frequency. From these observations we also see that, as has been reported for this protein and others at somewhat higher frequencies (e.g., ref 19), solvated lysozyme absorbs less strongly than the water that it displaces. The effect of the solute is thus to reduce the absorbance relative to that of the pure solvent.

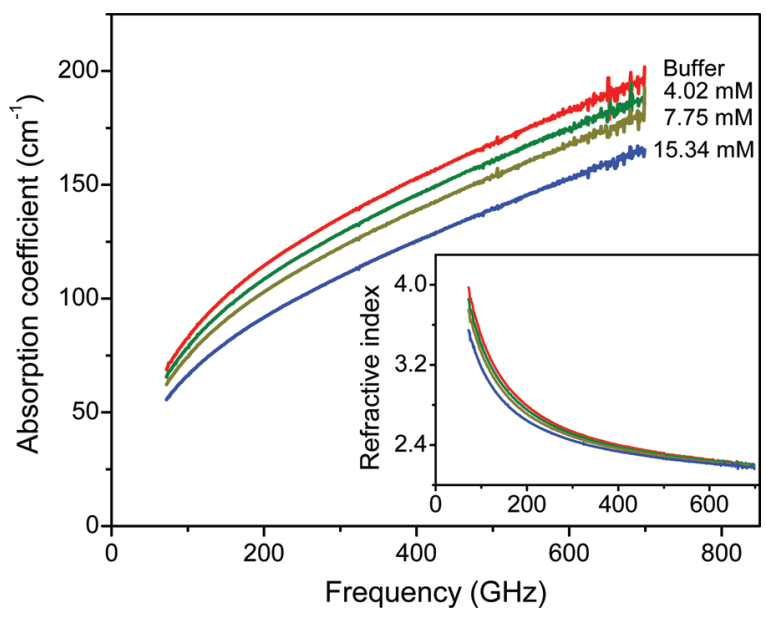

Figure 1. The gigahertz to terahertz absorption of both lysozyme solutions and a matched buffer blank increase monotonically with rising frequency. The refractive indices (inset) of lysozyme and buffer solutions, in contrast, decrease with increasing frequency. Both the absorbance and refractive index depend particularly strongly on frequency below $\sim 200 \mathrm{GHz}$. The data described here and in the following figures were collected at $25^{\circ} \mathrm{C}$ in $50 \mathrm{mM}$ sodium phosphate, $\mathrm{pH} 1.9$.

To compare our results with previous work, we first analyze the results using the assumption that the absorbance of the protein solution can be treated as a weighted average of the absorption of the solvent and of the protein. That is, the total absorption of the solvent, $\alpha_{\text {sol }}$ is given by $\alpha_{\text {sol }}=\sigma_{\text {lyz }} \cdot M_{\text {lyz }}+$ $\sigma_{\text {wat }} M_{\text {wat }}$, where $M_{\text {lys }}$ and $M_{\text {wat }}$ are the molarities, and $\sigma_{\text {lyz }}$ and $\sigma_{\text {wat }}$ are the "apparent" molar absorptions of the lysozyme and the solvent, respectively. In order to extract $\sigma_{\text {lyz }}$ from the experimentally observed $\alpha_{\text {sol }}$, we determined the molarity of the solvent in our samples volumetrically (see methods in Supporting Information). Using this value to determine the water's contribution to the overall absorbance of our sample, we can thus determine the apparent absorbance due to the protein alone. That is, by subtracting the "molarity-scaled" water baseline from the total solution absorption, we obtain $\sigma_{\mathrm{lyz}} M_{\mathrm{lyz}}$ and, in turn, the apparent molar extinction of the solvated protein, $\sigma_{\text {lys }}{ }^{19}$

Using the above approach to determine the apparent absorbance of solvated lysozyme, we obtain a striking result: over the entire frequency range, we have found that the absorption of the solvated protein is negative (Figure 2, left). This result, which appears quite "unphysical", has previously been rationalized as arising due to a hydration shell that is so tightly bound to the protein that it does not contribute to the absorption in this frequency range. ${ }^{18,19,32}$ If we assume that the absorbance of the protein is zero (realizing, of course, that in actual fact it may be greater than zero) at $220 \mathrm{GHz}$, the frequency at which the protein's absorbance is minimal, this "lost" solvent corresponds to $153 \pm 15$ water molecules in the hydration shell of each lysozyme molecule (equivalent to $0.19 \pm 0.02 \mathrm{~g}$ of water per gram of protein). We must note again, however, that although the assumption that the absorbance of lysozyme is never negative is clearly valid, the further assumption that the protein's minimum absorbance is precisely zero may also be incorrect, and thus the terahertz-defined hydration shell may contain more than this estimate of 153 water molecules.

Using the above analysis, we have measured the absorption spectrum of lysozyme in water at concentrations ranging from 


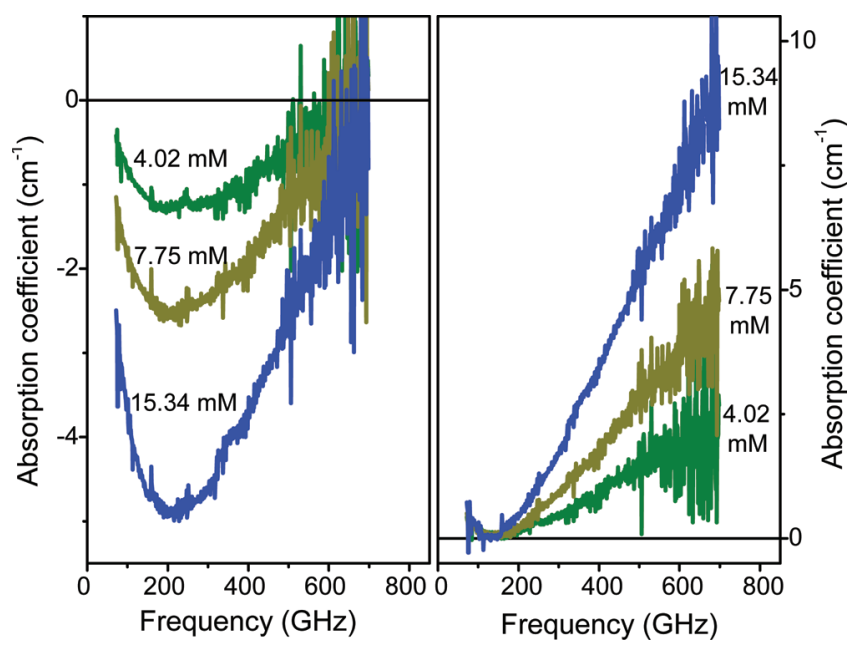

Figure 2. The absorption of solvated lysozyme provides a measure of the protein's low-frequency vibrational dynamics. (left) Absorbance of lysozyme (minus the absorbance of the relevant buffer blank) without any correction for the protein's hydration shell. This leads to negative absorption, which is unphysical. (right) Scaling of these spectra such that their absorbance minima reach zero suggests that $153 \pm 15$ water molecules in a hydration shell around each lysozyme no longer behave as if they were bulk solvent in terms of their gigahertz absorption.

$1.5 \%(\mathrm{w} / \mathrm{w})$, corresponding to $1.02 \mathrm{mM}$, to saturating, $26 \%$ (w/w) solutions corresponding to $15.34 \mathrm{mM}$. (Of note, the highest concentration spectrum is in good agreement with previous observations obtained using a free-electron laser source, ${ }^{18,19}$ albeit with much improved signal-to-noise and far higher spectral resolution, Figure S2, top, Supporting Information). These absorption spectra, which are characterized by a minimum at $\sim 220 \mathrm{GHz}$, retain quite similar shapes across this broad range of concentrations (Figure 2, right). Indeed, when these spectra are scaled by the concentration of lysozyme to produce molar absorbance spectra, we again observe "Beer's law" behavior in that the measured absorbance is proportional to the concentration of the protein (Figure S2, Supporting Information). This, in turn, suggests that the lysozyme's hydration shell is independent of protein concentration over the entire concentration range we have investigated. Given that only $5.0 \%$ of the water in even our highest concentration sample resides in this spectroscopically defined hydration shell, it is perhaps not surprising that the hydration shells on adjacent protein molecules do not interact significantly. This result contrasts with the nonBeer's law behavior reported previously for the protein lambda repressor by Havenith and co-workers, who attribute this behavior to interactions between the hydration shells of adjacent protein molecules. ${ }^{16,17}$ The report on non-Beer's law behavior, however, is only observed at protein concentrations well below the lowest concentrations that we can investigate with our spectrometer. Perhaps surprisingly given the above arguments, Havenith and coworkers do observe the Beer's law behavior at the higher protein concentrations employed here. Still, as lamda repressor is structurally distinct from lysozyme, and Havenith and co-workers performed their measurements at significantly higher frequencies than those employed here, direct comparison of the results of the two studies is difficult.

The above analysis assumes that the absorbance of a solution is a linear sum of the absorbance of the solvent and its solutes, an assumption that, as we have noted, may fail significantly in spectral regions for which the absorbance of the solvent changes very rapidly with frequency. With simultaneous measures of the absorbance and refractive index in hand, however, we can use effective medium models to extract the dielectric response of our solute, lysozyme, uncomplicated by this effect. Specifically, from knowledge of the absorbance and refractive index of lysozyme solutions we can compute the complex refractive index:

$$
n_{\text {sol }}^{*}(v)=n(v)+i K(v)
$$

where $v$ is frequency, $n$ is the refractive index of the solution, and $K$ is the extinction coefficient of the solution, which is related to the absorption coefficient, $\alpha$, by $K=c \alpha /(4 \pi v)$ with $c$ being the speed of light. From this we can calculate the complex dielectric function, $\varepsilon_{\mathrm{sol}}^{*}$, of the protein solution, which, in turn provides a complete description of the interaction of the solute with the incoming electromagnetic wave using the relationships:

$$
\begin{aligned}
& \varepsilon_{\mathrm{sol}}^{\prime}(v)=n^{2}(v)-\mathrm{K}^{2}(v)=n^{2}(v)-(c \alpha(v) / 4 \pi v)^{2} \\
& \varepsilon_{\mathrm{sol}}^{\prime \prime}(v)=2 n(v) \mathrm{K}(v)
\end{aligned}
$$

Taking these together, we obtain the complex dielectric response of the solution in which the real, $\varepsilon^{\prime}(v)$, and imaginary, $\varepsilon^{\prime \prime}(v)$, components represent the energy stored and energy dissipated per unit volume of the solution, respectively. Thus, from the above-described experimental measurements of $\alpha$ and $n$, we can determine the complete complex dielectric response of our protein solutions.

Protein solutions are, obviously, a mixture of water and protein, each of which exhibits its own complex dielectric constant, $\varepsilon_{\mathrm{wat}}^{*}$ and $\varepsilon_{\text {lyz }}^{*}$. These combine to define the complex dielectric constant of the solution, $\varepsilon_{\mathrm{sol}}^{*}$, which is the value determined from the experimental observables, $n$ and $\alpha$. Because the protein is orders of magnitude smaller than the wavelengths of the incoming electromagnetic radiation, the material can be treated as a homogeneous substance with an effective-dielectric function using the effective medium approximation of Bruggeman, ${ }^{26}$ which effectively treats both low and high concentration mixtures. (Note: the Maxwell Garnet and Wagner approximations ${ }^{24,25}$ are the low concentration limit of the Bruggeman approximation, which, in turn, is equivalent to the Hanai approximation). ${ }^{27}$ For a twocomponent mixture of a protein in water, and considering again the small sphere limit, we obtain the Bruggeman approximation for the complex dielectric constant of the solution as: ${ }^{23,25}$

$$
f_{\mathrm{p}} \frac{\varepsilon_{\mathrm{lyz}}^{*}-\varepsilon_{\mathrm{sol}}^{*}}{\varepsilon_{\mathrm{lyz}}^{*}+2 \varepsilon_{\mathrm{sol}}^{*}}+\left(1-f_{\mathrm{p}}\right) \frac{\varepsilon_{\mathrm{wat}}^{*}-\varepsilon_{\mathrm{sol}}^{*}}{\varepsilon_{\mathrm{wat}}^{*}+2 \varepsilon_{\mathrm{sol}}^{*}}=0
$$

with $f_{\mathrm{p}}$ being the volume fraction of the protein in the solution.

When we perform a Bruggeman effective-medium analysis, we find again the same striking result produced by the above, more naïve assumption of additive absorbances: $\varepsilon_{\mathrm{lys}}{ }^{\prime \prime}$, the imaginary (lossy) component of the complex dielectric constant of the protein, which is related to its absorption, is negative over the entire frequency range we have investigated. We correct for this by again assuming the protein entraps a hydration shell composed of $165 \pm 15$ water molecules per lysozyme $(0.21 \pm 0.02 \mathrm{~g}$ of water per gram of protein). This brings the imaginary component above zero such that, as expected for a nonconductor, $\varepsilon_{\text {lys }}{ }^{\prime \prime}$ extrapolates linearly to zero at zero frequency. Unlike the naive absorbance-based method used above and in prior literature, ${ }^{16-19}$ however, which estimate the size of the 
gigahertz-defined hydration shell by assuming that the protein's absorption falls to zero at its minima, this method of estimating the size of the hydration shell requires only the well-founded assumption that the protein's absorption falls to zero at zero frequency.

The value of 165 water molecules for the gigahertz-defined hydration shell corresponds to a submonolayer on the surface of the protein. Specifically, if we approximate lysozyme as a $\sim 2 \mathrm{~nm}$ diameter sphere, a solvent layer one molecule deep will contain 350 water molecules. Our value is also consistent with previous measurements made in the terahertz that suggest a (minimum) hydration shell of $\sim 173 \pm 40$ water molecules, ${ }^{19}$ and $<215$ water molecules as determined by a hydration measurement of the local relative humidity with a terahertz time-domain spectroscopy. ${ }^{32}$ This estimate differs, however, from earlier estimates of $259 \pm 51$ water molecules as defined by neutron and X-ray scattering $^{33,34}$ and $270 \pm 27$ from NMR measurements of the mobile, unfrozen water in frozen lysozyme solutions. ${ }^{35}$ These methods, however, employ definitions of the hydration shell that are physically distinct from that employed here. That is, our value of 165 water molecules corresponds to the number of waters that are bound strongly enough to the protein to act, in terms of their gigahertz absorption, as if they are protein and not bulk water. Crystallographic and NMR methods, in contrast, define the hydration shell in terms of fixed water density and the shell of water whose structure is so disrupted by the solute protein that it does not freeze. Given the significantly different structural and temporal physics underlying these distinct definitions of the hydration shell, this degree of matching is perhaps intriguing.

Seemingly in contrast with our results, molecular dynamics simulations and ${ }^{17} \mathrm{O}$ NMR relaxation dynamics suggest that, on average, the picosecond motions of the first hydration shell are only slowed down by a factor of $\sim 2$ (at room temperature) relative to the dynamics of bulk water. ${ }^{36}$ Again, however, gigahertz absorption spectroscopy and NMR relaxation spectroscopy use very different operational guidelines to define the hydration shell. Specifically, three significant differences likely contribute to this discrepancy. First, even the gigahertz-defined hydration shell is not "frozen" but instead consists of water molecules that are moving with the protein and not with the bulk solvent. That is, while they move more slowly than bulk water (i.e., they absorb less gigahertz radiation than bulk water does), they do still absorb in this frequency regime, thus suggesting that their picosecond motions are reduced but not eliminated. Second, as defined by gigahertz absorption, the hydration shell is less than one monolayer, and thus, if we were to average the dynamics over the entire first hydration shell, the mean change in water dynamics would be less than the change we observe. Third, NMR relaxation requires that the nuclear spin move through order of $\pi$ radians, motions that are much larger in magnitude than the likely subtle, small-magnitude harmonic motions that our approach is sensitive to. That is, NMR and gigahertz absorption are sensitive to motions occurring over very different "rotational" scales, which could easily account for the observed discrepancy.

To account for the hydration shell, which, again, behaves as if it is protein and not water at these frequencies, in the Bruggman approximation, we simply increase the excluded volume associated with the protein. Specifically, we scaled the volume-filling factor, $f_{\mathrm{p}}$, by 1.29 so that $\varepsilon_{\mathrm{lys}}{ }^{\prime \prime}$ extrapolates linearly to zero at zero frequency. Using this scaled filling factor and the measured $\varepsilon_{\text {wat }}^{*}$ and $\varepsilon_{\text {sol }}^{*}$, we can employ eq 3 to obtain the dielectric function of solvated lysozyme at each protein concentration we have

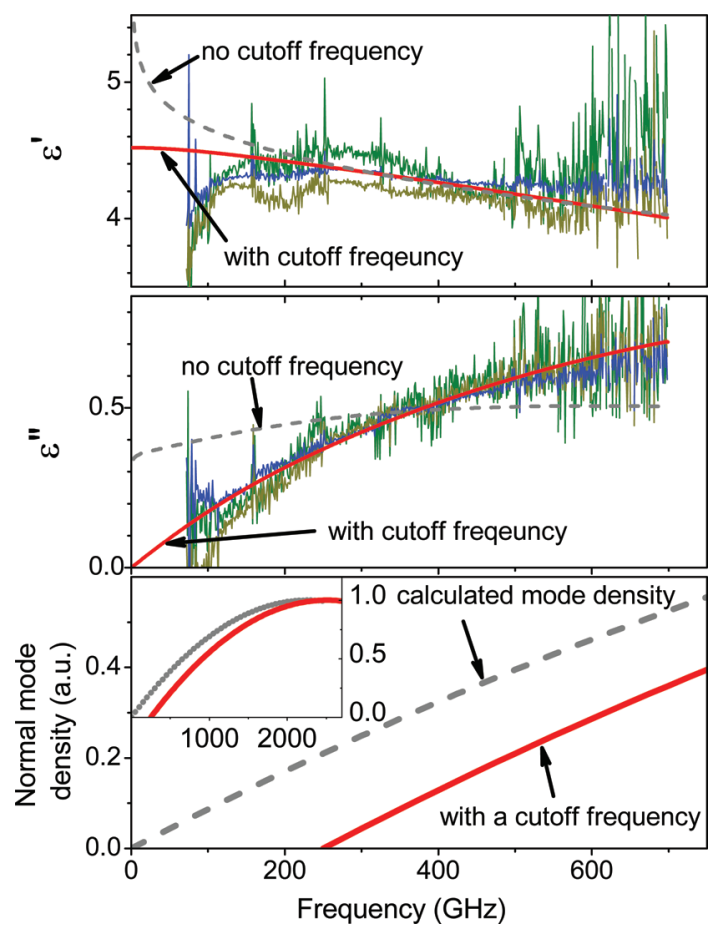

Figure 3. Integration of previously published, computational models of lysozyme's harmonic motions does not accurately recover the protein's experimentally observed complex dielectric spectra unless the modes are shifted to higher frequencies to introduce a low-frequency cutoff. Shown are the real (top panel) and imaginary (middle panel) components of the experimental dielectric spectra of lysozyme. Attempts to fit these spectra using calculated mode densities (bottom panel) taken from the literature (they are smoothed, and the inset to the bottom panel is the whole normal mode density for our simulation $)^{6}$ fail (dotted lines) unless we shift these calculated densities upward by $250 \mathrm{GHz}$ (red lines), producing a cutoff below which the mode density drops to zero. These fits involve two fitted parameters, the amplitude and the damping rate (see eq 4). The best fit damping rate, $594 \mathrm{GHz}$, indicates that the collective harmonic motions probed at these frequencies are overdamped, which presumably reflects strong coupling between these motions and the viscous solvent surrounding each protein molecule. The color scheme employed to denote the three data sets in each of the above panels is the same as that employed in Figure 2.

investigated (Figure 3). Upon doing so, we find these spectra are characterized by a rising imaginary component and a broad maximum of the real component. Thus, once again, the measured dielectric response extracted using the effective medium approximation does not depend on protein concentration, suggesting that the hydration shell is likewise independent of protein concentration.

As we have argued above, the complex dielectric response of solvated lysozyme over the gigahertz to terahertz spectral region should inform on the protein's collective, low-amplitude harmonic motions. The experimental results reported here thus provide an unprecedented opportunity to directly test computational models of the dynamics of this well-studied protein. To do so, we model the complex dielectric response of the protein as the summed response of a set of harmonic oscillators given by

$$
\varepsilon_{\mathrm{lyz}}^{*}(v)=\varepsilon_{\infty}+\int_{0}^{\infty} \frac{A f\left(v_{0}\right)}{\left(v_{0}^{2}-v^{2}\right)-i k_{\mathrm{F}}\left(v_{0}\right) \nu} \mathrm{d} v_{0}
$$


where $\varepsilon_{\infty}$ is the dielectric constant of lysozyme at frequencies far above those investigated here and $k_{\mathrm{F}}\left(v_{0}\right)$ is a frequency-dependent damping rate constant. In this equation the vibrational density of states, $f\left(v_{0}\right)$, weighted by the oscillator strength, $A$, provides a means of connecting our experimental results with theoretical models of lysozyme's harmonic motions. Specifically, we have extracted this function from a previous computational model of lysozyme's dynamics ${ }^{6}$ in order to ascertain whether the vibrational mode descriptions provided by this model are consistent with our observations.

To test these computational models against our experimental observations, we employ a frequency-independent damping rate, $k_{\mathrm{F}}$, and integrate all of the dielectric responses with resonance oscillation frequencies between zero and a high-frequency limit at several terahertz. In doing so, however, we find that these models fit our experimental data rather poorly (Figure 3, dashed line). For example, the best fit of the imaginary component of the experimentally observed dielectric response does not fall to zero at zero frequency (Figure 3, dashed curve). This would imply that the absorbance is not zero at zero frequency, which is physically impossible for a nonconductor such as lysozyme. In contrast, however, our experimental observations are reasonably well fit if we invoke a cutoff frequency, $v^{*}$, below which the vibrational mode density falls to zero (Figure 3, solid curves). Specifically, we find that by shifting the calculated mode density $250 \mathrm{GHz}$ toward higher frequencies (Figure 3, inset) and employing a damping rate, $k_{\mathrm{F}}$, of $594 \mathrm{GHz}$, we recapture both the real and imaginary components of the complex dielectric response of solvated lysozyme. Moreover, both this cutoff and this damping constant are physically reasonable. Specifically, the large value of the damping constant suggests that the large-scale motions probed in our studies are strongly coupled to the viscous solvent surrounding each lysozyme, which would lead to overdamping and a damping rate similar to or greater than the resonant frequency of the modes in question. The $250 \mathrm{GHz}$ cutoff likewise appears physically reasonable: given the protein's $\sim 2 \mathrm{~nm}$ diameter and the known speed of sound in a protein of $1960 \mathrm{~m} / \mathrm{s},{ }^{37}$ we calculate that the slowest harmonic motion would have a period of $\sim 0.8 \mathrm{ps}$, corresponding to a frequency cutoff of $\sim 200 \mathrm{GHz} .{ }^{19}$ We note, however, that all of the previous computational descriptions of the harmonic motions of lysozyme fail to observe cutoff; in all three the vibrational mode density drops off approximately linearly to zero. $^{6-8}$ It is unclear why this physically reasonable, herein experimentally verified cutoff is not apparent in these computational models. In contrast, simulations of the protein myogrobin suggest that there is a sharp cutoff at $\sim 150 \mathrm{GHz}\left(5 \mathrm{~cm}^{-1}\right) ;^{38}$ given that this protein is quite close in size to lysozyme, the similarity in the predicted myoglobin cutoff and the cutoff we observe experimentally appears compelling.

The experimentally observed cutoff that we report should not be taken to imply that proteins are motionless at time scales longer than $\sim 250 \mathrm{GHz}(\sim 0.6 \mathrm{ps})$. Indeed, abundant crystallographic and NMR studies have demonstrated that proteins are rife with conformational changes that contribute to their dynamic properties on time scales much longer than those probed in our experiments. ${ }^{11}$ Instead, our experimental results argue that, unlike these barrier-limited motions, the smaller-scale harmonic motions of proteins are characterized by a sharp cutoff, above which anharmonic, barrier-limited processes dominate the dynamics of biomolecules.

\section{CONCLUSION}

We have developed a frequency-domain terahertz spectroscopy with phase-sensitive detection and high dynamic range. The spectrometer with orders of magnitude improved signal-to-noise allows us to accurately measure strong absorption materials, such as the aqueous samples employed here. We have measured the gigahertz to terahertz complex dielectric response of solvated lysozyme and used the Bruggeman effective-medium approximation to define the size of the protein's hydration shell and connect our experimental observations to previous computational models of the protein's collective, low-amplitude harmonic motions. We find that, independent of the protein's concentration, the dielectric-defined hydration shell of lysozyme contains $165 \pm 15$ water molecules, a value closely consistent with previous estimates based on other experimental approaches. Our experiments also raise questions regarding the validity of computational models of the low-frequency harmonic motions of this protein. That is, modeling the complex dielectric response as the summed response of a set of damped harmonic oscillators in order to directly connect our experimental observations with previous computational models of lysozyme dynamics, we find relatively poor agreement. This agreement can be improved, however, by introducing a low-frequency cutoff at $250 \mathrm{GHz}$ below which our experiments suggest the density of vibrational modes drops to zero. The absence of such a cutoff in the equivalent computational models of lysozyme would, in turn, suggest that more refined computational tools may be needed in order to accurately model the low-frequency collective vibrational modes of proteins.

\section{ASSOCIATED CONTENT}

S Supporting Information. The materials, solution preparation, absorption and refractive index measurements, and molar absorption of solvated biomolecules. This material is available free of charge via the Internet at http://pubs.acs.org/.

\section{AUTHOR INFORMATION}

\section{Corresponding Author}

Vinh@itst.ucsb.edu

\section{ACKNOWLEDGMENT}

The authors acknowledge the helpful advice and support provided by Prof. Mark Sherwin. This work was supported by a grant from the W. M. Keck Foundation.

\section{REFERENCES}

(1) Modig, K.; Liepinsh, E.; Otting, G.; Halle, B. J. Am. Chem. Soc. 2003, 126, 102-114.

(2) Daniel, R. M.; Dunn, R. V.; Finney, J. L.; Smith, J. C. Annu. Rev. Biophys. Biomol. Struct. 2003, 32, 69-92.

(3) Karplus, M.; Kuriyan, J. Proc. Natl. Acad. Sci. U.S.A. 2005, $102,6679-6685$.

(4) de Groot, B. L.; Hayward, S.; van Aalten, D. M. F.; Amadei, A.; Berendsen, H. J. C. Proteins: Struct., Funct., Genet. 1998, 31, 116-127.

(5) Brooks, B.; Karplus, M. Proc. Natl. Acad. Sci. U.S.A. 1985, 82, 4995-4999.

(6) Markelz, A.; Whitmire, S.; Hillebrecht, J.; Birge, R. Phys. Med. Biol. 2002, 47, 3797-3805.

(7) Levitt, M.; Sander, C.; Stern, P. S. J. Mol. Biol. 1985, 181, 423-447. 
(8) van Vlijmen, H. W. T.; Karplus, M. J. Phys. Chem. B 1999, 103, 3009-3021.

(9) Fischer, B. M.; Walther, M.; Jepsen, P. U. Phys. Med. Biol. 2002, 47, 3807-3814.

(10) Zhang, C.; Durbin, S. M. J. Phys. Chem. B 2006, 110, 23607-23613.

(11) Kempf, J. G.; Loria, J. P. Cell Biochem. Biophys. 2002, 37, $187-211$.

(12) Mchaourab, H. S.; Kalai, T.; Hideg, K.; Hubbell, W. L. Biochemistry 1999, 38, 2947-2955.

(13) Ghosh, S.; Mandal, U.; Adhikari, A.; Dey, S.; Bhattacharyya, K. Int. Rev. Phys. Chem. 2007, 26, 421-448.

(14) Globus, T. R.; Woolard, D. L.; Khromova, T.; Crowe, T. W.; Bykhovskaia, M.; Gelmont, B. L.; Hesler, J.; Samuels, A. C. J. Biol. Phys. 2003, 29, 89-100.

(15) Schwartz, S. D.; Schramm, V. L. Nat. Chem. Biol. 2009, 5, $551-558$.

(16) Ebbinghaus, S.; Kim, S. J.; Heyden, M.; Yu, X.; Gruebele, M.; Leitner, D. M.; Havenith, M. J. Am. Chem. Soc. 2008, 130, 2374-2375.

(17) Ebbinghaus, S.; Kim, S. J.; Heyden, M.; Yu, X.; Heugen, U.; Gruebele, M.; Leitner, D. M.; Havenith, M. Proc. Natl. Acad. Sci. U.S.A. 2007, 104, 20749-20752.

(18) Xu, J.; Plaxco, K. W.; Allen, S. J. Protein Sci. 2006, 15, $1175-1181$.

(19) Xu, J.; Plaxco, K. W.; Allen, S. J. J. Phys. Chem. B 2006, 110, 24255-24259.

(20) Fukasawa, T.; Sato, T.; Watanabe, J.; Hama, Y.; Kunz, W.; Buchner, R. Phys. Rev. Lett. 2005, 95, 197802.

(21) Markelz, A. G.; Roitberg, A.; Heilweil, E. J. Chem. Phys. Lett. 2000, 320, 42-48.

(22) Whitmire, S. E.; Wolpert, D.; Markelz, A. G.; Hillebrecht, J. R.; Galan, J.; Birgey, R. R. Biophys. J. 2003, 85, 1269-1277.

(23) Choy, T. C. Effective medium theory: principle and applications; Clarendon Press: Oxford, 1999; p 7.

(24) Maxwell Garnett, J. C. Philos. Trans. R. Soc. London 1904, 203, 385-420.

(25) Wagner, K. W. Arch. Elektrotech. (Berlin) 1914, 2, 371-387.

(26) Bruggemann, D. A. G. Ann. Phys. Leipzig 1935, 24, 636-679.

(27) Hanai, T. Colloid Z. 1960, 171, 23-31.

(28) Wei, Y. Z.; Kumbharkhane, A. C.; Sadeghi, M.; Sage, J. T.; Tian, W. D.; Champion, P. M.; Sridhar, S.; McDonald, M. J. J. Phys. Chem. 1994, 98, 6644-6651.

(29) Yokoyama, K.; Kamei, T.; Minami, H.; Suzuki, M. J. Phys. Chem. B 2001, 105, 12622-12627.

(30) Oleinikova, A.; Sasisanker, P.; Weingartner, H. J. Phys. Chem. B 2004, 108, 8467-8474.

(31) Kindt, J. T.; Schmuttenmaer, C. A. J. Phys. Chem. 1996, 100, 10373-10379.

(32) Knab, J.; Chen, J.-Y.; Markelz, A. G. Biophys. J. 2006, 90, 2576-2581.

(33) Svergun, D. I.; Richard, S.; Koch, M. H. J.; Sayers, Z.; Kuprin, S.; Zaccai, G. Proc. Natl. Acad. Sci. U.S.A. 1998, 95, 2267-2272.

(34) Merzel, F.; Smith, J. C. Proc. Natl. Acad. Sci. U.S.A. 2002, 99, 5378-5383.

(35) Kuntz, I. D. J. Am. Chem. Soc. 1971, 93, 514-516.

(36) Mattea, C.; Qvist, J.; Halle, B. Biophys. J. 2008, 95, 2951-2963.

(37) Pfeiffer, H.; Heremans, K. Chem. Phys. Lett. 2002, 361, 226-230.

(38) Yu, X.; Leitner, D. M. J. Phys. Chem. B 2003, 107, 1698-1707. 